Relato de Caso

Case Report

Eliana Maria Gradim Fabron 1,2

Kelly Cristina Alves Silvério²

Giédre Berretin-Felix ${ }^{2}$

Eduardo Carvalho Andrade ${ }^{2}$

Polyana Ferreira Salles²

Pâmela Aparecida Medeiros Moreira² Alcione Ghedini Brasolotto²

Descritores

Voz

Treinamento da voz

Fisiologia

Qualidade da voz

Idoso

Keywords

Voice

Voice training

Physiology

Voice quality

Elderly

Endereço para correspondência: Eliana Maria Gradim Fabron

Avenida Hugyno Muzzi Filho, 737,

Bairro Mirante, Marília (SP), Brasil,

CEP: 17525-000.

E-mail: elianaf@marilia.unesp.br

Recebido em: Outubro 20, 2017

\section{Terapia vocal para idosos com progressão de intensidade, frequência e duração do tempo de fonação: estudo de casos}

\author{
Voice therapy for the elderly with progression \\ of intensity, frequency, and phonation time: \\ case reports
}

\begin{abstract}
RESUMO
Este trabalho pretende verificar o efeito imediato e em médio prazo na voz e na laringe de dois idosos submetidos à terapia vocal intensiva com progressão de intensidade e frequência vocais e de duração do tempo de fonação. Dois idosos (um homem, 79 anos e uma mulher, 82 anos) com queixa vocal e características de presbilaringe, realizaram 12 sessões de terapia vocal intensiva com progressão de intensidade e frequência da voz e duração do tempo de fonação, durante três semanas. Para analisar o efeito terapêutico foram realizadas avaliações perceptivoauditivas e acústicas da voz, de tempo máximo de fonação (TMF), de autorreferência da qualidade de vida em voz e do comportamento laríngeo nos momentos pré, imediatamente após e um mês depois do processo de terapia vocal. Os valores da maioria das medidas resultantes dessas avaliações indicaram mudanças positivas imediatamente após a terapia vocal para os idosos. Observou-se redução das medidas perceptivoauditivas de desvio da qualidade vocal e diminuição das medidas de perturbação e ruído do sinal acústico, o que indica melhora na voz. Houve elevação da frequência fundamental e, aumento do TMF, além de autorreferência de melhor qualidade de vida em voz. A avaliação das imagens laríngeas não mostrou diferença consistente. Após um mês do término da terapia alguns parâmetros pioraram em relação ao momento pós-imediato, mesmo assim, permaneceram melhores em relação ao momento pré-terapia. Desta forma os resultados da proposta terapêutica são promissores e seus efeitos devem ser pesquisados em estudos clínicos controlados para verificar sua eficácia em idosos.
\end{abstract}

\begin{abstract}
This study aims to verify the immediate and medium-term effects of an intensive voice therapy, with progression of vocal intensity and frequency and phonation time, on the voice and larynx of two elderly. A 79-year-old male and an 82-year-old female with vocal complaints and presbylarynx characteristics underwent 12 sessions of intensive voice therapy, with progression of voice intensity and frequency and phonation time, for three weeks. To analyze the therapeutic effects, the following assessments were performed pre-, post-immediately, and one month after voice therapy: auditory-perceptual analyses of the voice, acoustic analysis, and evaluation of maximum phonation time (MPT), self-reference voice-related quality of life, and laryngeal behavior. Most results of these measurements indicated positive changes immediately after voice therapy. There was reduction in the measures of vocal quality deviation, perturbation, and noise-to-harmonic ratio pre-, post-immediately, and one month after voice therapy, which indicates vocal improvement. There was increase in fundamental frequency, maximum phonation time and self-reference voice-related quality of life. Assessment of the laryngeal images showed no consistent difference. One month after voice therapy, worsening of some results was observed compared with the post-immediate assessment, but improvements were maintained in relation to the initial evaluation. The results of this therapeutic proposal are promising, and their effects should be investigated in controlled clinical trials to verify their efficacy.
\end{abstract}

Trabalho realizado no , Departamento de Fonoaudiologia, Faculdade de Odontologia de Bauru - USP Bauru (SP), Brasil.

${ }^{1}$ Departamento de Fonoaudiologia, Faculdade de Filosofia e Ciências - UNESP - Marília (SP), Brasil

${ }^{2}$ Departamento de Fonoaudiologia, Faculdade de Odontologia de Bauru - USP - Bauru (SP), Brasil.

Fonte de financiamento: Fundação de Amparo à Pesquisa do Estado de São Paulo - FAPESP. Processo número: 2016/12836-4.

Conflito de interesses: nada a declarar. 


\section{INTRODUÇÃO}

A terapia vocal para o idoso tem sido de grande interesse para pesquisadores e profissionais da área pela grande procura desta população por tratamentos visando à melhora na sua qualidade de vida com melhor desempenho vocal ${ }^{(1)}$. Estudos sobre as modificações fisiológicas da laringe dos idosos; a prevalência de problemas vocais; a sua qualidade vocal e o envolvimento da voz nas condições psicossociais do idoso foram reportados em artigo de revisão bibliográfica ${ }^{(1)}$. Além disso, foram desenvolvidas pesquisas buscando evidência científica de terapia vocal para os idosos ${ }^{(2-10)}$. A metodologia utilizada nesses estudos, bem como, a quantidade de participantes e intervenção aplicada variou muito. Dentre as intervenções para a reabilitação vocal ressaltam-se estudos com propostas fechadas de terapia como os Exercícios de função vocal $(\mathrm{EFV})^{(2-4)}$, o método Lee Silverman Voice Treatment (LSVT) adaptado para idosos ${ }^{(5-6)}$; o método Phonation Resistence Training Exercise (PhoRTE) ${ }^{(7)}$; o Programa Vocal Cognitivo $(\mathrm{PVC})^{(8)}$; a Terapia Vocal para idosos (TVI) ${ }^{(9)}$ e ainda, uma proposta terapêutica, baseada na fisiologia do exercício, tendo a estimulação elétrica como adjuvante ${ }^{(10)}$. $O$ treinamento com base na fisiologia do exercício deve ser refletido a partir de seis princípios: adaptação, sobrecarga, especificidade, progressão e reversibilidade. O princípio da sobrecarga refere-se ao aumento do uso habitual de um sistema e a progressão é a utilização do aumento da carga continuamente para que se alcance a mudança habitual desejada ${ }^{(11)}$, sendo que pode-se destacar um dos estudos com idosos que apoiou-se nos princípios da progressão e sobrecarga, pois a partir da medida máxima da intensidade, da frequência da voz e do tempo máximo de fonação, foram propostos exercícios com a carga de $60 \%, 70 \%$ e $80 \%$ desta medida inicial, visando ao alcance cada vez maior do desempenho desses parâmetros (intensidade e frequência da voz e duração do tempo de fonação) na terapia vocal para o idoso $^{(10)}$. O sucesso do tratamento da alteração vocal no idoso depende de mudanças da intensidade vocal, do uso muscular, do suporte respiratório e, sobretudo da manutenção das mudanças provocadas no sistema fonatório. Diante dos princípios da fisiologia do exercício e das necessidades contemporâneas da comunicação dos idosos, a terapia vocal baseada principalmente no princípio da progressão tem potencial para favorecer o equilíbrio vocal em idosos.

A partir do conhecimento da fisiologia do exercício, o objetivo deste estudo é verificar o efeito imediato e em médio prazo na voz e na laringe de dois idosos submetidos à terapia vocal intensiva com progressão de intensidade e frequência vocais e de duração do tempo de fonação.

\section{MÉTODO}

Os casos apresentados são os resultados preliminares de um estudo aprovado pelo Comitê de Ética em Pesquisa de uma Instituição (CAEE:56422916.9.0000.5417). Os participantes assinaram o Termo de Consentimento Livre e Esclarecido, consentindo com a realização e divulgação da pesquisa e seus resultados.

\section{Participantes}

Um homem (S1) e uma mulher (S2) (79 e 82 anos, respectivamente) com queixas relacionadas à voz que surgiram no período do envelhecimento, além de exame laringológico compatível com a presbilaringe, com a presença de arqueamento de pregas vocais e/ou saliência de processos vocais durante a respiração e/ou fenda fusiforme à fonação ${ }^{(12)}$. S1 apresentou boa saúde geral, vivia com a esposa, realizava atividades físicas semanalmente e participava de atividades sociais. S2 também apresentava boa saúde geral, vivia sozinha e visitava os familiares nos finais de semana. Além disso, apresentavam compreensão das atividades propostas e não tinham histórico de câncer na região de cabeça e pescoço, doenças neurológicas ou neuromotoras e também não eram fumantes ou ex-fumantes.

\section{Intervenção}

Foi elaborada uma Proposta de terapia vocal com progressão de intensidade e frequência da voz e de duração do tempo de fonação, baseando-se na proposta de LaGorio, Carnaby-Mann e Crary $(2010)^{(10)}$. A proposta consiste numa série de exercícios vocais desenvolvidos com objetivo de aumentar gradativamente a resistência vocal e expandir a capacidade fonatória. A partir de uma medida base de emissão vocal calculam-se metas fonatórias com cada participante. Para tanto, mede-se o maior tempo máximo de fonação (TMF), a intensidade e a extensão vocal de fonação sustentada. Essa medida é realizada por três vezes e a linha de base é considerada a medida maior sendo esta, utilizada para dar início aos passos da proposta.

As metas para cada indivíduo foram calculadas para desenvolver o treino com $60 \%, 70 \%$ e $80 \%$ de cada medida de base propondo, desta forma, uma hierarquia no trabalho terapêutico. A terapia foi desenvolvida com a emissão de vogais, "a" e "i" e a consoante " $m$ ", contagem de números, frases e leitura de textos ${ }^{(10)}$. Outras vogais puderam ser utilizadas conforme a facilidade do participante, principalmente as vogais "ô" e "ó".

No início o participante deve sustentar a fonação de uma vogal por $60 \%$ do TMF do valor de base até um bom desempenho vocal, depois passar para $70 \%$ e $80 \%$. Da mesma forma, são adicionadas fonações com outra vogal e consoante. O mesmo processo é realizado para o trabalho com a intensidade e extensão vocal. Conforme o indivíduo melhora seu desempenho vocal com a emissão sustentada de "a", "i” e "m", são inseridos exercícios com palavras, frases e conversação. Após uma sequência de exercícios, em que o participante cumpra todas as metas, outras medidas de base devem ser realizadas para nova proposta com novas metas. As medidas que o indivíduo alcança são anotadas tanto para um controle terapêutico como para incentivá-lo a alcançar o que foi proposto.

Cada passo desta proposta deve ser executado com o controle do terapeuta sobre a qualidade de emissão em relação ao apoio respiratório, à estabilidade, à intensidade e à qualidade vocal. O paciente deve ser levado a prestar atenção no trabalho que está realizando, fazendo o controle proprioceptivo e auditivo da fonação. Desta forma o desenvolvimento terapêutico é individualizado, pois depende das conquistas diárias na terapia, 
mas o processo de progressão é padronizado seguindo sempre as metas de $60 \%, 70 \%$ e $80 \%$ dos valores de base.

Esta proposta terapêutica foi desenvolvida durante três semanas, por quatro dias em cada semana, com duração de uma hora cada. Cada participante era incentivado a conquistar suas metas, sendo que as medidas eram controladas por meio de cronômetro, decibelímetro e um aplicativo de apresentação de frequência fundamental $\left(\mathrm{F}_{0}\right)^{1}$. Durante toda sessão era oferecida água aos participantes. A partir da sessão que o paciente conseguia fazer emissões com boa qualidade vocal, ele era incentivado a realizar os exercícios em casa, mais uma vez ao dia. A sequência de exercícios foi fornecida por escrito e conforme havia o treino de lista frases ou poemas na sessão terapêutica, o material era entregue ao paciente para fazer parte de sua tarefa diária. No final das 12 sessões cada participante foi orientado sobre a importância de continuar a fazer os exercícios diariamente.

\section{Avaliações}

Para verificar o efeito da proposta terapêutica foram realizadas: avaliações perceptivoauditiva, acústica e de TMF, autoavaliação de qualidade de vida relacionada à voz, além de avaliação laríngea por meio da nasoendoscopia e da telelaringoscopia. Tais avaliações foram realizadas nos momentos Pré-terapia (Pré), imediatamente após a terapia (PI) e após um mês do término da terapia (PT).

Para a avaliação perceptivoauditiva e a análise acústica foram realizadas gravações das vozes dos participantes nos momentos PRÉ, PI e PT. As gravações foram realizadas numa sala acusticamente tratada utilizando o gravador digital Marantz modelo PMD660 e microfone Sennheiser e845. O microfone foi posicionado a $4 \mathrm{~cm}$, lateralmente, da boca dos sujeitos, que permaneceram sentados em frente ao avaliador. Os participantes foram orientados a emitirem a vogal "a" sustentada o máximo de tempo possível, mantendo a frequência e a intensidade habituais. Cada gravação foi editada mantendo-se aproximadamente três segundos de emissão, recortando-se o início e o final do sinal acústico para que, respectivamente, o ataque vocal e a instabilidade no final da emissão, não interferissem na análise dos dados.

Para a realização da avaliação perceptivoauditiva, o grupo das três gravações da vogal sustentada, que foram realizadas nos momentos Pré, PI e PT, de cada participante, foram randomizadas e apresentadas aos três fonoaudiólogos treinados para esta atividade e que não participaram de qualquer outro procedimento do estudo. Foi utilizada uma escala visual analógica de $100 \mathrm{~mm}$, pelos parâmetros grau geral, rugosidade, soprosidade, tensão, pitch e loudness em que o menor valor do desvio encontrado relaciona-se com a melhor qualidade do mesmo e, quanto maior o valor, maior o seu desvio (protocolo CAPE- $\mathrm{V}^{(13)}$ ).

As gravações editadas foram analisadas pelo Software MDVP-Multi-Dimentional Voice Program (KAY-PENTAX), nos parâmetros de: frequência fundamental $\left(\mathrm{F}_{0}\right)$, Jitter Percent (Jitt), $\mathrm{vF}_{0}=$ coeficiente de variação da frequência fundamental; $S h \mathrm{~dB}=$ Shimmer in $\mathrm{dB} ; \mathrm{vAm}=$ coeficiente de variação de

\footnotetext{
Aplicativo Da Tuna Lite (Play Store)
}

amplitude e Noise-to-Harmonic Ratio (NHR). O TMF foi medido utilizando o MDVP pela emissão sustentada de "a", "s" e "z" em pitch e loudness habituais. Para a extração da medida foi considerado o maior tempo de três emissões para a vogal e para cada consoante.

Para a autoavaliação foi aplicado o protocolo $\mathrm{QVV}^{(14)}$ por um fonoaudiólogo, colaborador, que não participou do processo terapêutico, nos momento Pré, PI e PT.

A avaliação laringológica foi realizada por um médico otorrinolaringologista no momento da avaliação inicial para inclusão dos participantes do estudo; o mesmo profissional realizou os exames nos momentos pós-terapia. Um outro avaliador experiente, cego em relação aos momentos de avaliação, comparou as imagens dos vídeos do exame laringológico a fim de verificar possíveis mudanças entre os momentos Pré, PI e PT. Para esta avaliação os vídeos dos exames foram editados retirando-se a identificação dos participantes e mantendo apenas as imagens de visualização das pregas vocais durante a respiração e a fonação. Os três arquivos, resultantes da edição dos exames de cada participante, foram randomizados para que fossem comparados os momentos PI com o Pré, o PT com o Pré e PT com o PI e apresentados ao avaliador.

Os parâmetros avaliados na telelaringoscopia foram: arqueamento das pregas vocais, saliência de processos vocais, fechamento glótico; na nasolaringoscopia foram: fechamento glótico, contrição medial de pregas vestibulares e constrição anteroposterior durante a emissão da vogal. Os critérios de avaliação foram: houve melhora, piora ou nenhuma mudança das imagens.

\section{Análise dos dados}

Os resultados foram analisados de forma descritiva. Para verificar a confiabilidade entre as respostas dos três fonoaudiólogos que realizaram a avaliação perceptivoauditiva, foi aplicado o teste de correlação de Pearson, cujos resultados indicaram correlações positivas $(\mathrm{p}<0,05)$ entre os avaliadores 1 e $2(\mathrm{r}=0,477)$; 1 e 3 $(\mathrm{r}=0,522) ; 2$ e $3(\mathrm{r}=0,623)$. Diante deste resultado, foi feita a média dos três valores para a discussão.

\section{RESULTADOS}

A tabela 1 apresenta os resultados da comparação entre as avaliações perceptivoauditiva, de análise acústica e dos TMFs, do Protocolo QVV dos momentos Pré, PI e PT.

Em relação à avaliação perceptivoauditiva, os dados representam a média dos valores assinalados pelos três juízes fonoaudiólogos, em milímetros (mm). Os dados da autoavaliação, do QVV, são apresentados em porcentagem nos domínios global, físico e socioemocional.

Os resultados da avaliação perceptivovisual dos vídeos dos exames laríngeos revelaram que, das 36 respostas dos parâmetros analisados, 47,22\% indicou não ter havido mudança nos aspectos laríngeos; $27,77 \%$ delas apontou piora e 13,99\% melhora nesses aspectos; não houve condições de análise da imagem em 11,11\%. 
Tabela 1. Medidas das avaliações perceptivoauditiva, acústica, de Tempos Máximos de Fonação e do protocolo Qualidade de Vida em Voz, realizadas nos momentos pré (Pré), pós-imediato (PI) e após um mês de terapia (PT)

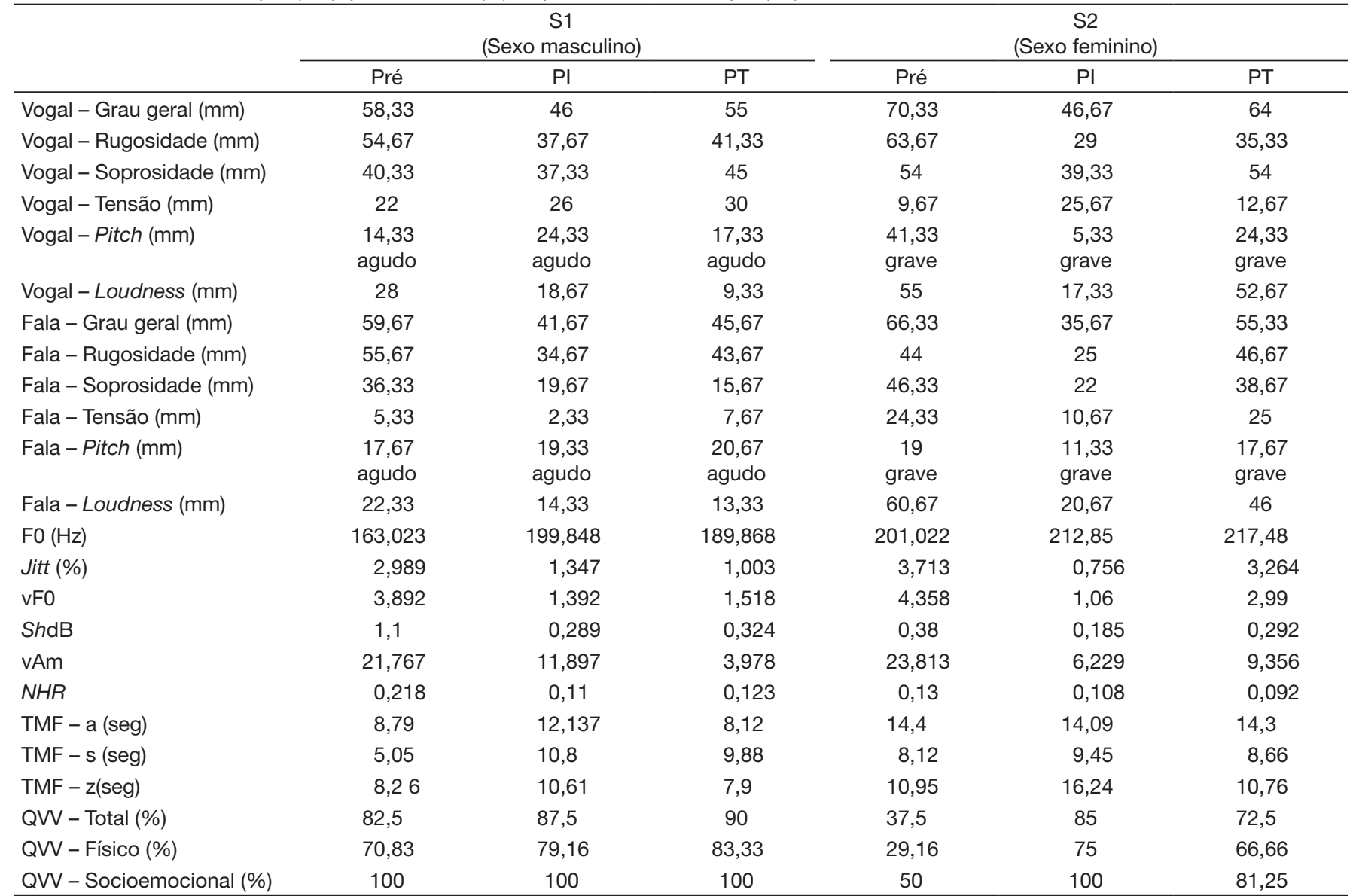

Legenda: $\mathrm{mm}=$ milímetro; $\mathrm{seg}=$ segundos; $\mathrm{F}_{0}=$ frequência fundamental; Jitt = Jitter Percent; $\mathrm{vF0}=$ coeficiente de variação da frequência fundamental; ShdB = Shimmer in dB; vAm = coeficiente de variação de amplitude; NHR = Noise-to-Harmonic Ratio; TMF = Tempo Máximo de Fonação; QVV = Protocolo de Qualidade de Vida em Voz

\section{DISCUSSÃO}

As avaliações realizadas foram capazes de demonstrar o efeito, imediato e em médio prazo, da terapia vocal com progressão de intensidade e frequência de voz e de duração do tempo de fonação na voz e na laringe nos dois idosos. Em geral, pode-se afirmar que houve melhora na qualidade vocal e na autoavaliação de qualidade de vida em voz imediatamente após a realização das 12 sessões da terapia, num período de três semanas. Os resultados das medidas realizadas um mês após a terapia mostraram particularidades dos participantes.

No que se refere à avaliação perceptivoauditiva da emissão sustentada da vogal "a" e da fala espontânea, na comparação dos resultados entre os momentos Pré, PI e PT, de forma geral, observa-se uma tendência de melhora imediata em todos os parâmetros avaliados (PI) e, após um mês da finalização do processo terapêutico (PT), houve uma piora no grau de desvio da qualidade vocal no momento PT.

Para considerar a correspondência entre o grau corte e os desvios moderado e intenso, numa escala visual analógica para o desvio da rugosidade foram adotados os valores entre 28,5 e 59,5 e entre 59,5 e 100 , respectivamente; e da soprosidade, os valores entre 33,5 e 52,5, para desvio moderado e, entre 52,5 e 100 para intenso ${ }^{(15)}$. Os resultados encontrados entre os dois idosos que participaram da proposta terapêutica, em relação à emissão da vogal, apresentavam desvio da rugosidade de grau moderado (S1) e intenso (S2), no momento Pré, alcançaram grau de desvio moderado no PI e, no PT, o grau moderado, mas com valores aumentados, entretanto menores que os encontrados no momento Pré. Da mesma forma, com desvio moderado, foram observados os valores encontrados nos diversos momentos em relação à soprosidade e, neste parâmetro, o momento PT foi elevado um pouco mais que em Pré para S2.

Pelo fato de a avaliação do grau geral de impacto vocal acompanhar os maiores resultados julgados, os valores deste parâmetro seguiram a mesma tendência. A percepção do desvio de tensão apontou que os seus valores, ainda que pequenos, aumentaram após a terapia vocal, fato provocado provavelmente pela progressão de intensidade que se desenvolve durante a realização dos exercícios. Outro estudo de casos apontou a manutenção da tensão de grau leve na qualidade vocal ${ }^{(8)}$. No parâmetro pitch os desvios encontrados demonstram a tendência do uso de uma voz um pouco mais agudizada para $\mathrm{S} 1$ e menos grave para S2 após esta proposta terapêutica, fato 
provocado pelo trabalho progressivo com a frequência da voz. As avaliações da loudness na emissão da vogal "a" mostraram grande diminuição do desvio no PI para os dois participantes. No momento PT, S1 apresentou o melhor resultado de sua intensidade vocal, mostrando que conseguiu mantê-la após ter finalizado o processo terapêutico, mas S2 não conseguiu a mesma performance, concordando com outro estudo que verificou a falta de manutenção da intensidade vocal no momento pós $\operatorname{tardio}^{(9)}$. A melhora encontrada na avaliação perceptivoauditiva foi também encontrada nos poucos artigos que descreveram este tipo de instrumento para verificar o efeito terapêutico na voz do idoso ${ }^{(4,8-9)}$.

A avaliação perceptivoauditiva de gravações de fala espontânea apresentou a mesma tendência que a encontrada na de vogais, isto é, resultados de PI melhores que em Pré, mas decréscimo deste ganho de qualidade no PT. O mesmo resultado foi encontrado após a finalização da proposta com o programa Terapia Vocal de Idosos (TVI) $)^{(9)}$.

Em relação às medidas acústicas observou-se a elevação de $\mathrm{F}_{0}$ nos momentos PI e PT em relação ao Pré, dado que apóia a percepção dos juízes sobre a variação do pitch dos participantes. Um estudo ${ }^{(6)}$ que analisou o efeito imediato da LSVT aplicada em dois idosos encontrou o mesmo resultado no momento pós-terapia. Na proposta terapêutica aqui apresentada os parâmetros trabalhados também foram o TMF, a intensidade e extensão vocal, apresentando uma diferença importante que é o controle da hierarquização das metas a serem conseguidas a partir de uma medida de base. Esta hierarquização apoia-se na fisiologia do exercício ${ }^{(11)}$. O mesmo comportamento vocal foi observado em pesquisa ${ }^{(8)}$ em que além dos objetivos de elevar a intensidade, aumentar a extensão vocal, utilizou exercícios de relaxamento cervical, firmeza glótica e sons nasais, entretanto com foco no processo cognitivo de desenvolvimento. A elevação da $\mathrm{F}_{0}$ é uma demonstração da reação muscular durante a fonação, a qual é reforçada com o trabalho em intensidade elevada.

As medidas de perturbação (jitter, $\mathrm{vF}_{0}$, shimmer e vAm) e de ruído (NHR) mostraram grande decréscimo nos seus valores, principalmente na comparação entre o Pré e o PI, sendo que a maior diferença encontrada foi nos valores de vAm dos dois participantes. Medidas de perturbação e ruído do sinal acústico foram demonstradas em três trabalhos ${ }^{(3,6,8)}$ com mudanças positivas e significativas em dois deles ${ }^{(6,8)}$ sugerindo que as propostas do $\mathrm{LSVT}^{(6)}$ e do $\mathrm{PVC}^{(8)}$ ofereceram um suporte para maior equilíbrio na qualidade de vibração de pregas vocais e suposta adequação do fechamento glótico. As medidas acústicas $\mathrm{vF}_{0}$ e vAm, parecem ser medidas que sofreram maiores modificações nos dois casos relatados aqui. A terapia vocal intensiva, com progressão de intensidade e de frequência da voz e de duração do tempo de fonação, favoreceu o equilíbrio da emissão sonora, com maior estabilidade da produção sonora também ao longo da emissão.

A proposta terapêutica aqui apresentada tem como um dos elementos importantes a hierarquia do trabalho com o TMF. A avaliação destas medidas, tanto da vogal "a" como das consoantes "s" e "z", mostrou uma reação importante do desempenho dos participantes na sustentação das emissões, principalmente na comparação dos resultados entre Pré e PI, apontando maior eficiência na dinâmica respiratória. Resultados dessa natureza foram encontrados em outros estudos ${ }^{(6,8)}$. Entretanto, no PT houve uma nova diminuição no TMF dos idosos. Os resultados do momento PT apontam para a necessidade de uma abordagem diferente junto aos idosos na tentativa de mostrar a importância da mudança de hábitos no seu dia-a-dia, com a realização dos exercícios de forma mais atenta.

As mudanças na qualidade vocal que ocorreram após o processo terapêutico proposto foram semelhante para os dois participantes. Embora não tenham sido observadas modificações nos aspectos laríngeos para a maioria dos parâmetros analisados, ocorreram modificações vocais perceptíveis auditivamente, além de modificações acústicas. A proposta terapêutica com progressão de intensidade e frequência da voz e de duração de tempo de fonação tem o potencial de fortalecer a musculatura intrínseca da laringe, melhorar a vibração de mucosa das pregas vocais e equilibrar a coordenação de fluxo aéreo, que refletem em redução da rouquidão, soprosidade e, consequentemente, grau geral de desvio vocal.

O desenvolvimento da percepção de uma voz mais equilibrada proporcionada pelos efeitos do treinamento diário e a generalização desses efeitos para a fala encadeada e conversa espontânea permitiram que parte dos benefícios observados no pós-terapia imediato permanecessem até o período tardio de acompanhamento. $\mathrm{O}$ fato do parâmetro tensão ter aumentado na vogal no momento pós-imediato é um aspecto positivo, uma vez que a voz do idoso com presbilaringe apresenta características de baixa intensidade e diminuição da extensão vocal, conforme relatado em revisão de literatura ${ }^{(1)}$ e, ao ser feito treinamento para torná-la mais forte, aumenta a tensão, o que condiz com uma voz mais equilibrada para o idoso. Isso é reforçado pelo resultado de redução do desvio de loudness, pois a voz dos participantes tornou-se mais forte, tanto na vogal como na fala encadeada.

Os valores do QVV podem indicar o quanto um problema de voz ocasiona impacto na sua qualidade de vida. Os resultados do S1 nos domínios Total (DT), Físico (DF) e Socioemocional (DSE) mostram que a sua alteração vocal não impunha um problema na sua qualidade de vida. Assim mesmo, é possível observar que os resultados dos momentos PI e PT aumentaram (DT e DF) ou se mantiveram no valor máximo (DSE).

O S2 referiu grande melhora na sua qualidade de vida relacionada ao seu problema vocal, pois no momento Pré os domínios Total e Físico apontavam um grande impacto da voz na sua vida e no Domínio Socioemocional, um problema moderado. No momento PI os valores alcançados chegaram a apontar para um valor de normalidade nesta relação, entretanto no PT, S2 relatou sentir uma piora, sem, contudo, alcançar o mesmo nível de Pré. O período de três semanas de terapia intensiva foi capaz de proporcionar a percepção de um excelente relacionamento entre a qualidade de voz alcançada e a qualidade de vida dos participantes. Dentre os estudos que utilizaram a autoavaliação para verificar a eficiência de uma proposta de terapia vocal para idosos ${ }^{(2,3,7,9-10)}$, um deles ${ }^{(2)}$ mostrou pelos resultados do Protocolo QVV que a terapia vocal proposta baseada no Exercício de Função Vocal (EFV) havia proporcionado significante melhora na percepção que os sujeitos tinham do impacto da voz na sua vida, entretanto os valores após o processo terapêutico ainda apresentaram valores médios 
de 77, indicando um impacto moderado da voz na qualidade de vida. Outro estudo( ${ }^{(7)}$ que comparou o método PhoRTE com o Exercício de Função Vocal (EFV) e com outro grupo controle, utilizando o QVV, mostrou que os dois grupos que realizaram a terapia vocal durante quatro semanas, apresentaram melhoras significativas nos escores do protocolo. Os valores médios do DT encontrado pelos pesquisadores para estes dois grupos nas medidas pré-terapia já apresentaram-se altos $(88,5$ e 80,8$)$ e melhoraram após a intervenção $(96,9$ e 87,5). Na pesquisa com o Terapia Vocal para Idosos (TVI), a autora encontrou que os valores do QVV foram melhores tanto na terapia intensiva, como na sua aplicação em sessões espaçadas ${ }^{(9)}$. Esse é um dos poucos estudos que avaliou os mesmos resultados um mês após sua finalização e observou a manutenção dos resultados ${ }^{(9)}$. A avaliação tardia do efeito da proposta terapêutica é importante para levantar informações como o paciente está se relacionando com os efeitos da terapia vocal. Os protocolos de autoavaliação são instrumentos sensíveis para verificar o quanto o trabalho vocal com o idoso favorece a sua qualidade de vida. Com o aumento do envelhecimento da população, um grande desafio é a manutenção da qualidade de vida dos idosos, sendo um dos aspectos a serem considerados, a manutenção da sua comunicação efetiva e, com ela a boa qualidade vocal. As pesquisas realizadas em busca de uma proposta terapêutica para o idoso ${ }^{(1-10)}$ demonstraram a sua importância ao alcançarem que a melhora vocal provocada pelos exercícios vocais diminuem o impacto negativo de sua voz na qualidade de vida.

$\mathrm{Na}$ avaliação das imagens dos exames laríngeos, telelaringoscopia e nasolaringoscopia, a maioria das respostas dos diversos critérios analisados apontou que não houve mudança. Resultados de avaliação tardia para verificar a eficiência de propostas terapêutica para idosos são escassos na literatura. Em um estudo de caso ${ }^{(6)}$ foram descritas as avaliações de pós tratamento duas semanas depois de sua finalização e encontrou o fechamento glótico total para um dos sujeitos (senhora de 61 anos) e no outro, a redução na fenda glótica (senhor de 88 anos). O exame laríngeo do trabalho citado, foi realizado pela estroboscopia e analisado por meio de medidas numéricas utilizando software apropriado para isso, com edição de um videoclipe de um segundo ${ }^{(6)}$. A diferença de metodologia utilizada não favorece a comparação dos resultados, entretanto, o melhor resultado encontrado pelos autores foi em reação ao participante mais jovem, da mesma forma que o verificado no estudo ora apresentado. A condição do fechamento glótico foi alvo de um estudo $^{(5)}$ que encontrou resultados diversos no exame laríngeo após o processo terapêutico proposto, apontando que dependendo do tamanho da fenda glótica, e da atrofia das pregas vocais, o alcance do fechamento glótico total, por meio de exercícios vocais, pode ser mais difícil. A relação entre a qualidade vocal e a condição laríngea no idosos deve ser considerada em estudos futuros. Novos estudos devem ser realizados com objetivos de melhor compreender a relação entre as condições do fechamento glótico, da constrição de região supraglótica e vibração das pregas vocais com a qualidade vocal resultante das propostas de terapia vocal para idosos.

A quantidade de sessões e a duração em semanas ou meses, de realização do processo terapêutico descrito nos estudos apresentados na literatura variaram de quatro sessões realizadas numa média de cinco meses ${ }^{(2)}$; seis sessões, uma vez na semana ${ }^{(3)}$ ou seis sessões com exercícios preestabelecidos em cada uma delas ${ }^{(8)}$; oito semanas de exercícios realizados duas vezes ao dia em casa $^{(4)}$ e os estudos estruturados de forma intensiva com 13 sessões, sendo 3 semanas e 5 sessões por semana (duas sessões de ambientação com a proposta) ${ }^{(10)}$ ou ainda aqueles estruturados com 16 sessões em quatro semanas de processo terapêutico ${ }^{(6-7,9)}$.Todas as propostas terapêuticas mostraram melhoras na qualidade vocal dos participantes, ou nos relatos de autoavaliação. Para a prática fonoaudiológica é importante a realização de investigações que tragam evidências científicas para o tratamento vocal do idosos (EFV, LSVT, PhoRTE, CPV, TVI), mas que também busquem o seu resultado ao longo do tempo (TVI) ${ }^{(9)}$.

Esta proposta terapêutica, intensiva, baseada na fisiologia do exercício, com a hierarquização de metas a serem atingidas dos parâmetros de intensidade e de frequência da voz e de duração do tempo de fonação, foi determinada para ser realizada em três semanas, com 12 sessões de uma hora cada, por se entender que poderia facilitar a adesão do paciente à terapia, ser um período menor e capaz de promover mudanças fisiológicas na emissão vocal. Nestes dois casos apresentados, foi evidente que a proposta proporcionou melhorias na qualidade vocal e nas medidas de autoavaliação. As avaliações realizadas após um mês de finalização das terapias (PT) mostraram, com exceção de algumas medidas do TMF, resultados melhores que as do momento Pré.

\section{COMENTÁRIOS FINAIS}

A proposta de terapia vocal intensiva com progressão de intensidade e de frequência vocais e de duração para idosos trouxe benefícios para a voz dos participantes mostrando em todas as avaliações realizadas que após um mês do final das sessões terapêuticas tal benefício se manteve na grande maioria dos resultados.

Os resultados das avaliações dos dois participantes mostraram a mesma tendência entre os momentos Pré, PI e PT, entretanto é possível observar resultados particulares. Houve diferença entre os resultados do QVV dos participantes, com melhora consistente no momento PI e resultados divergentes no PT. Entre as medidas de análise acústica foi observado aumento da frequência fundamental dos momentos PI e PT em relação ao Pré, e diminuição nos valores das medidas de perturbação e de ruído do sinal sonoro no PI e, no PT, valores menores que aqueles encontrados no Pré. Os resultados do TMF das três emissões foram variados, porém apresentaram manutenção ou elevação dos tempos em PI e manutenção dos mesmos em PT, sendo que em dois dos resultados houve o valor de PT menor que o encontrado no momento Pré.

Os resultados desta proposta de terapia vocal para idosos são promissores e seus efeitos devem ser verificados em estudos clínicos controlados, com um número maior de participantes e com grupo controle para que se possa verificar sua eficácia. 


\section{AGRADECIMENTOS}

Agradecemos o recurso concedido da FAPESP (processo $n^{\circ}$ 2016/12836-4), sem o qual não seria possível a realização da pesquisa. Aos fonoaudiólogos Millena Maria Ramalho Matta Vieira, Jhonatan da Silva Vitor, Angélica Emygdio da Silva Antonetti e Cínthia Procópio da Silva pela contribuição no desenvolvimento do estudo.

\section{REFERÊNCIAS}

1. Oates JM. Treatment of dysphonia in older people: the role ofthe speech therapist. Curr Opin Otolaryngol Head Neck Surg. 2014;22(6):477-86. http://dx.doi.org/10.1097/MOO.0000000000000109. PMid:25250623.

2. Berg EE, Hapner E, Klein A, Johns MM 3rd. Voice therapy improves quality of life in age-related dysphonia: a case-control study. J Voice. 2008;22(1):704. http://dx.doi.org/10.1016/j.jvoice.2006.09.002. PMid:17070009.

3. Sauder C, Roy N, Tanner K, Houtz DR, Smith ME. Vocal function exercises for presbylaryngis: a multidimensional assessment of treatment outcomes. Ann Otol Rhinol Laryngol. 2010;119(7):460-7. http://dx.doi. org/10.1177/000348941011900706. PMid:20734967.

4. Kaneko M, Hirano S, Tateya I, Kishimoto Y, Hiwatashi N, Fujiu-Kurachi $\mathrm{M}$, et al. Multidimensional analysis on the effect of vocal function exercises on aged vocal fold atrophy. J Voice. 2015;29(5):638-44. http://dx.doi. org/10.1016/j.jvoice.2014.10.017. PMid:25944293.

5. Mau T, Jacobson BH, Garrett CG. Factors associated with voice therapy outcomes in the treatment of presbyphonia. Laryngoscope. 2010;120(6):11817. PMid:20513037.

6. Lu FL, Presley S, Lammers B. Efficacy of intensive phonatory-respiratory treatment (LSVT) for presbyphonia: two case reports. J Voice. 2013;27(6):786. e11-23. http://dx.doi.org/10.1016/j.jvoice.2013.06.006. PMid:24119640.

7. Ziegler A, Verdolini Abbott K, Johns M, Klein A, Hapner ER. Preliminary data on two voice therapy interventions in the treatment of presbyphonia. Laryngoscope. 2014;124(8):1869-76. http://dx.doi.org/10.1002/lary.24548. PMid:24375313.

8. Nemr K, Souza GV, Simões-Zenari M, Tsuji DH, Hachiya A, Cordeiro GF, et al. Cognitive Vocal Program applied to individuals with signals presbylarynx: preliminary results. CoDAS. 2014;26(6):503-8. http://dx.doi. org/10.1590/2317-1782/20142014108. PMid:25590914.

9. Godoy JF. Efetividade do programa terapia vocal para idosos nas formas convencional e intensiva [tese]. Bauru: Faculdade de Odontologia de Bauru, Universidade de São Paulo; 2016.

10. Lagorio LA, Carnaby-Mann G, Crary MA. Treatment of vocal fold bowing using neuromuscular electrical stimulation. Arch Otolaryngol Head NeckSurg. 2010;136(4):398-403. http://dx.doi.org/10.1001/archoto.2010.33. PMid:20403858.

11. Weineck J. Treinamento ideal. São Paulo: Manole, 2003

12. Pontes P, Brasolotto AG, Behlau M. Glottic characteristics and voice complaints in the elderly. J Voice. 2005;19(1):84-94. http://dx.doi. org/10.1016/j.jvoice.2004.09.002. PMid:15766853.

13. Behlau M. Consensus Auditory- perceptual Evaluation of Voice (CAPE-V), ASHA 2003. Refletindo sobre o novo. Rev Soc Bras Fonoaudiol. 2004;9(3):187-9.

14. Gasparini G, Behlau M. Quality of life: validation of the Brasilian version of the Voice-Related Quality of Life (V-RQOL) measure. J Voice. 2009;23(1):7681. http://dx.doi.org/10.1016/j.jvoice.2007.04.005. PMid:17628396.

15. Baravieira PB, Brasolotto AG, Montagnoli AN, Silvério KCA, Yamasaki R, Behlau M. Análise perceptivo-auditiva de vozes rugosas e soprosas: correspondência entre a escala visual analógica e a escala numérica. CoDAS. 2016;28(2):163-7. http://dx.doi.org/10.1590/2317-1782/20162015098. PMid:27191880.

\section{Contribuição dos autores}

EMGF participou no delineamento do estudo, na realização das terapias, na análise dos resultados e na elaboração do manuscrito; KCAS participou no delineamento do estudo, na avaliação perceptivo auditiva e das imagens do exame laríngeo, na análise dos resultados e na elaboração do manuscrito; GBF participou no delineamento do estudo, na análise dos resultados e na revisão crítica do manuscrito; ECA participou na realização dos exames laríngeos, bem como no diagnóstico otorrinolaringológico inicial; PFS participou na coleta dos dados, na alimentação da planilha de dados e na discussão dos resultados; PAMM participou na coleta dos dados, na alimentação da planilha de dados e na discussão dos resultados; AGB participou no delineamento do estudo, na análise dos resultados e na elaboração do manuscrito. 